\title{
Dismenorrea en la adolescencia
}

\author{
Juan Carlos Rojas Martínez*, Claudia Patricia Robles Roa*, Néstor Andrés Rojas Martínez
}

\section{RESUMEN}

OBJETIVO: Determinar la prevalencia de la dismenorrea (DSM) en nuestra institución, estudiar sus características en la adolescencia, determinar las implicaciones de la DSM en el ausentismo escolar.

DISEÑO: Estudio abierto, prospectivo controlado con placebo. Se encuestaron 600 adolescentes. Ingresaron 141 con DSM primaria entre 12 y 23 años de edad. Se formaron tres grupos según el tratamiento, así: a 21 medios comunes tradicionales. El segundo grupo: 35 se les administró un placebo y el tercer grupo: 85 recibieron Fenoprofeno Cálcico, dosis inicial de 600 mg luego una cápsula T.I.D. por tres días, por tres ciclos menstruales. En el cuarto ciclo no se suministró tratamiento. El dolor se cuantificó mediante escala visual análoga, aplicada al momento de tomar la primera dosis y a los 15, 30, 120, 180 minutos, en los días siguientes se pidió evaluar el dolor, cuantificar el tiempo a sentir mejoría y la presencia de efectos secundarios.

RESULTADOS: EI 27\% de estudiantes padecían DSM: 21 DSM secundaria, no se incluyeron 141 presentaban DSM primaria. EI $70 \%$ tenían entre 15 y 17 años, el $61 \%$ padecían DSM desde la menarquia. El promedio de evolución de la DSM fue de 2 años. La clasificación del dolor obtenida por la escala visual análoga, fue: Grado I (leve) 27.66\%, Grado II (moderado) 54.61\% y Grado III (Severo) $17.63 \%$. EI $83 \%$ de las adolescentes se automedicaba, de ellas no lograban mejoría el $78 \%$. Sólo el 16,9\% consultaron al médico. 33/141 tenían antecedentes de DSM en su familia. Se describen las asociaciones clínicas entre DSM y ciclo menstrual en la adolescencia. Así como las características del dolor como síntoma de DSM.

Los valores promedios obtenidos en las escalas visuales demuestran que después de la primera dosis, existe una diferencia, muy significativa, en favor del fenoprofeno cálcico, la cual se mantuvo a todo lo largo del período de observación, con una $p<0=0.01($ es decir su nivel de confianza es del 99\%); al compararlo con el placebo y con el medicamento natural. En los ciclos de evaluación, el fenoprofeno mejora a más del $90 \%$ de las adolescentes rápidamente, antes de 15 minutos.

CONCLUSIONES: La DSM, para nuestra institución constituye la tercera causa de prevalencia, después del parasitismo intestinal y los problemas de visión. Es más frecuente durante la menstruación y no premenstrual como es descrito. Las escalas visuales establecieron la intensidad del dolor, siendo leve y moderado en el 83,2\% de las adolescentes en la literatura el resultado es inverso. Los ensayos infieren al dolor incapacitante en el $3 \%$ de los casos, en nuestro estudio el $7 \%$ de las adolescentes se incapacitan 2 y 3 días y el $12 \%$ un día, retrasando su proceso educativo. Las adolescentes refieren síntomas asociados a la DSM, en la literatura se menciona su hallazgo a edades mayores. EI $\mathbf{4 1 \%}$ se automedica, generando patologías iatrogénicas y consumistas, acostumbrándose a ingerir medicamentos sin considerar los riesgos futuros, sólo mejora el $36 \%$, es otra pauta de lo mal orientado del tratamiento; aún más, sólo el $17 \%$ consultan al médico. El promedio general obtenido por la mediana estadística demuestra que el uso correcto de el AINE mejora al $96 \%$ de las adolescentes y a partir del tercer ciclo disminuye la intensidad y frecuencia de la DSM y de los síntomas asociados. Concluimos que debe permitirse desde el aula una orientación adecuada respecto al tratamiento de la DSM, agrupándola en los programas de salud de los colegios con servicio médico.

PALABRAS CLAVES: Dismenorrea, menstruación, dolor, automedicación, adolescencia.

\section{SUMMARY}

OBJECTIVES: Determination of the prevail of DSM in our educational institutions. Study of both particular DSM characteristics during adolescence. Determination of DSM implications with regard to absenteeism from school.

DESIGN: Open prospectively conducted study controllable with placebo. Six hundred adolescent women were surveyed. A total of 141 adolescent women were included in this study, aging between 12 to 23 years. The study was conducted prospectively in three groups in accordance with medication to be administered, as follows: the first group of 21 adolescent women was treated with common traditional methods; the second group of 35 was treated with placebo and the third group of 85 adolescent students received Calcium Phenoprophen; an initial dose of $600 \mathrm{mg}$ and during the two subsequent days, they received a TID capsule. Medications were administered during three menstrual consecutive cycles. During their fourth consecutive cycle, adolescent women were not treated. Pain was then quantizated by means of $100 \mathrm{~mm}$ long visual scales provided; visual scales were applied 15,30,120 and 180. During subsequent days, adolescents were asked to appraise and inform on both valuation of their menstrual pain and the time elapsed before pain was relieved.

RESULTS: Of 27\% were affected with DSM: 21 DSM secondary dysmenorrhea. Adolescents were excluded from the study. 141 patients presenting primary. $70 \%$ of adolescents were aged between 15 and 17 years. $61 \%$ suffered DSM from their menarche. Evolution of DSM 
was two years in average. Following were the results obtained from pain scales: $27.66 \%$ Grade I (light pain); 54.61\% Grade II. (Moderate pain) and $17.63 \%$ Grade III (severe pain). This is the $83 \%$ of them had been self-medicating. $78 \%$ of these adolescents did not succeed with pain relievers; only the $16.9 \%$ consulted physicians. $33 / 141$ adolescents had familiar DSM antecedents. The article describe thas clinical associations between painjns DSM and menstrual cycles a adolescence. So like thas painjns caracteristics symptom the DSM.

Averaged values of pain obtained from the visual scales, after the first dose, favoring the calcium phenoprophen; this difference was maintained along the whole observation period: $p<0=0.01$, (meaning that its faith level is $99 \%$ ) when compared with placebo and natural medicine. During the valuation cycles, it was observed that within 15 minutes, phenoprophen quickly relieved paint to more than $90 \%$ of the adolescents.

DISCUSSION: Institutionally, DSM represents the third cause of students prevail, besides intestinal parasitism and vision troubles. Pain more often appears during menstruation, and not the premenstural period as it has been described. Graduation of pain by means of visual scales, allowed the determination of its intensity, being light-related and moderate in the $83.2 \%$ of the studied adolescents; a the literature inverted results. Reviewed tests state that disabling pain is present in $3 \%$ of reported cases; our study revealed that $7 \%$ of adolescent women were disabled during 2 or 3 days and $12 \%$ one day, delaying their educational process.

Together with this, adolescents described symptoms associated with DSM a literature where weakened state and low academic performance are found in older women. $41 \%$ of adolescents in our study medicated generating another iatrogenic and consumerist pathologies; young persons being in the habit of self-medication of any kind of drugs at their expense and the future risks. Only relieved pain to $36 \%$ of the adolescents studied; this reflects the bad orientation given to DSM treatment, still more when only the $17 \%$ of them consult the physician. Obtained general averaged results by using middle statistics ratio showed that AINS relieves pain in 96\% of treated adolescents; furthermore, starting from the third treatment cycle, both intensity and frequency of menstrual pain diminished and so any other clinical related manifestation. We can conclude that it may be allowed, from the school, and adequate orientation regarding treatment of this pathology by joining it with health programs in the different schools having a medical service.

KEY WORDS: Dysmenorrhea, menstruation, pain, self-medication, adolescence.

\section{Introducción}

Etimológicamente la palabra dismenorrea proviene del griego dus (mal dolor), mên (mes) y rhein (fluir). Y define cualquier tipo de dolor relacionado con la menstruación; es un síntoma básico, ya que causa una importante incapacidad laboral o académica, de automedicación y de consulta médica. Adquiere igualmente importancia al ser el principal síntoma subjetivo de múltiples patologías ginecológicas que pueden tener interés por sus variables consecuencias.

Se acepta que lo presentan el 30 a $50 \%$ de las pacientes postpuberales; en el $10 \%$ será motivo de incapacidad de sus funciones normales, la dismenorrea o menstruación dolorosa constituye un problema de gran importancia social y económica. En Estados Unidos ha presentado la pérdida de 140 millones de horas de trabajo por año; en Francia, cerca de 30 millones, en Colombia no existen estudios de este tema.

Existe una cierta correlación con la pubertad precoz, el tabaco y la nuliparidad, mientras que los factores como la talla, el peso y la regularidad del ciclo menstrual no parece que ejerzan influencia alguna sobre el proceso.

Clásicamente se ha dividido en Dismenorrea Primaria: Cuando aparecía desde la primera menstruación, y secundaria, cuando lo hacía después de un período indefinido de menstruaciones no dolorosas. Atendiendo a la causa, se ha clasificado en dismenorrea esencial: aquella en la que no se observa una causa y dismenorrea orgánica: Cuando, por el contrario, se demuestra un motivo que la produce, tiene como causas principales: la endometriosis estenosis cervical, la enfermedad pélvica inflamatoria, el ovario poliquístico, laiomiomas, pólipos y adherencias uterinas; malformaciones congénitas del sistema Mülleriano y adenomiosis.
Para comprender mejor la fisiopatología de la dismenorrea esencial se requiere el conocimiento previo de la fisiología de la contracción uterina durante el ciclo menstrual y los distintos mecanismos que están implicados en su regulación (3-5).

Debemos distinguir los distintos patrones contráctiles que se observan en las diferentes fases del ciclo genital $y$ en las pacientes dismenorreicas.

Se considera que en el período folicular, fundamentalmente de acción estrogénica, las contracciones uterinas son de escasa intensidad y frecuentes (20-60/min), manteniendo un tono basal entre 10 y $30 \mathrm{~mm} \mathrm{Hg}$. Durante la ovulación aumenta de forma significativa el tono basal (sobre los $50 \mathrm{~mm} \mathrm{Hg}$ ), disminuyendo la frecuencia de las contracciones. En la fase lútea desciende el tono basal (10 a $30 \mathrm{~mm} \mathrm{Hg}$ ) y la amplitud de las contracciones aumenta, disminuyendo su frecuencia. En la fase menstrual se observa tono basal prácticamente nulo, aumentando la intensidad (hasta $100 \mathrm{~mm} \mathrm{Hg}$ ) y la amplitud (cada 1-4 min.) de la contracción uterina.

En la paciente dismenorreica se observa un aumento importante del tono basal, superior a $10 \mathrm{~mm} \mathrm{Hg}$, polisistolia e hipersistolia que pueden llegar a $150 \mathrm{~mm} \mathrm{Hg}$. Las contracciones son irregulares y en ocasiones no llegan a descender al tono base ya de por sí alterado, según se refiere anteriormente. El aumento del tono y la intensidad de las contracciones impide la correcta irrigación del miometrio uterino, en el que aparecen fenómenos isquémicos que son capaces de producir dolor (6-9).

Desde Hipócrates existen teorías para explicar la fisiopatología del proceso, tal es la teoría obstructiva, sostiene que la dificultad en la apertura del cérvix para expulsar, generaría contracciones anómalas capaces de producir dolor, de hecho se observa que luego del parto 
se presenta mejoría clínica del proceso, por aumentar la dilatación del orificio cervical; pero la mejoría también se ve en mujeres sometidas a cesárea antes del inicio del trabajo de parto, lo que hace pensar que existen otros factores distintos a la dilatación, pueden ser nerviosos u hormonales.

Los factores hormonales se relacionan por los cambios que se presentan después de cualquier tipo de parto y por el hecho de que la dismenorrea es característica del ciclo ovulatorio; se desconoce la relación entre hormonas y prostaglandinas; Pikles las ha asociado con la dismenorrea y postuló que se producirían en exceso, o bien su metabolismo sería deficitario y, por tanto, se acumularían aumentando su acción. El mecanismo de acción sería aumentar la contractibilidad uterina por elevar los niveles de calcio libre intracelular y disminuir el dintel de sensibilidad nerviosa sensitiva a los estímulos mecánicos y químicos. En resumen las molestias de la dismenorrea esencial son consecuencia de la respuesta uterina normal a las prostaglandinas, el dolor se debe a niveles elevados de prostaglandina F2 alfa; (10) así mismo son responsables del florido cuadro clínico que acompaña al síndrome de tensión premenstrual (11-13).

La participación nerviosa en la regulación de la contracción uterina se demuestra por la presencia de terminaciones nerviosas adrenérgicas y colinérgicas en el miometrio, igualmente por la mejoría que presentan mujeres sometidas a una simpatectomía presacra. El mecanismo de acción es un aumento del tono simpático que produciría un aumento de la contractibilidad uterina.

Desde siempre se ha relacionado la dismenorrea con una personalidad de tipo histérico, actualmente se sostiene que los factores psicológicos podrían actuar como moduladores de la dismenorrea, pero nunca como origen de ésta. La modulación respondería a transtornos psicosexuales o bien a fenómenos ambientales que afectarían la personalidad de la paciente.

En resumen:

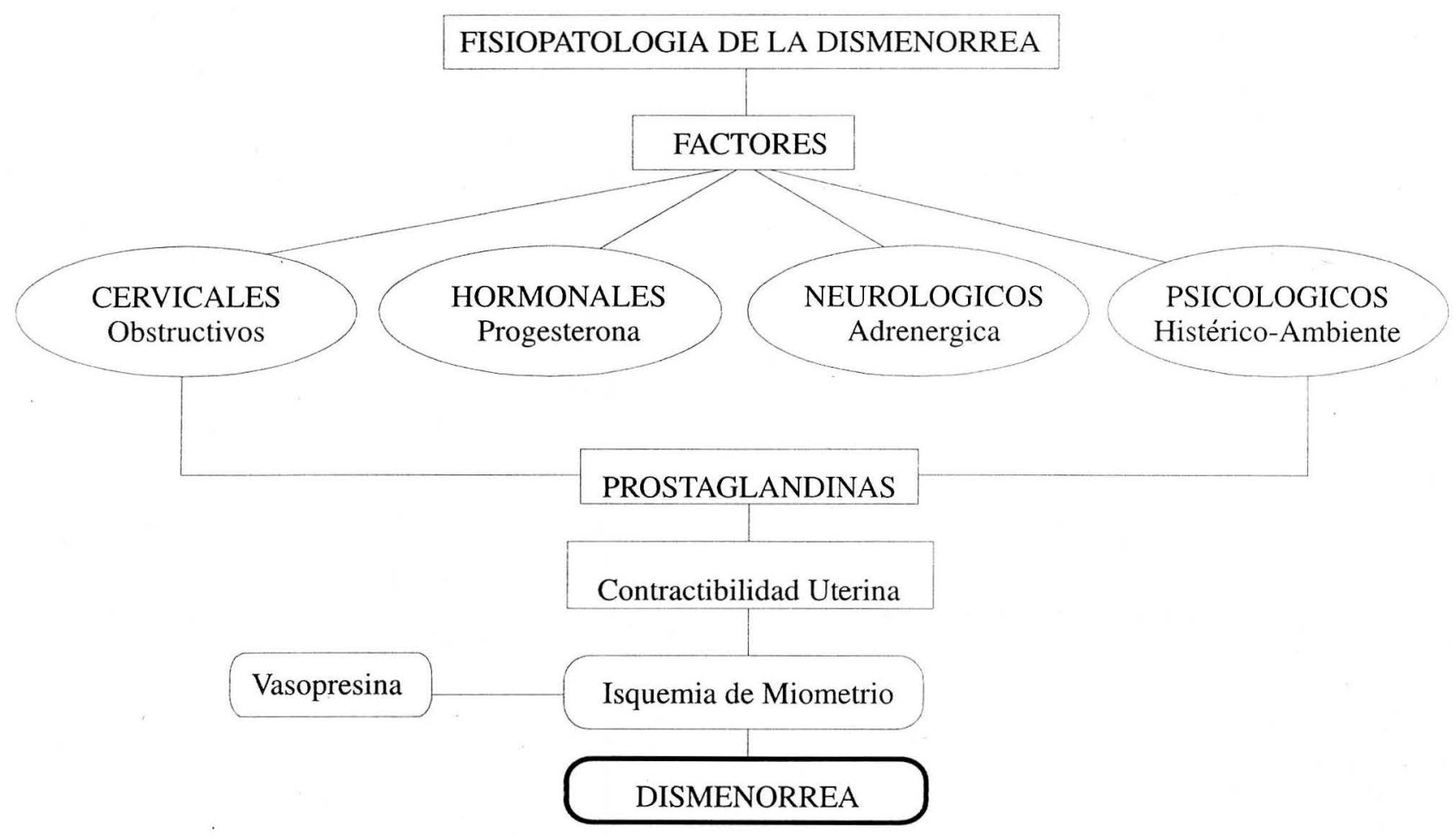

En cuanto a la fisiopatología de la dismenorrea orgánica, se deberá establecer por la enfermedad causal, así entonces, la endometriosis causaría dismenorrea por la distensión de los quistes endometriósicos al aumentar su contenido durante el período menstrual y por lo tanto a la fibrosis peritoneal secundaria (15-16). Las estenosis orgánicas, así como las malformaciones uterinas o vaginales, o el dispositivo intrauterino, (17) generan dolor por el mismo mecanismo que las dismenorreas esenciales (23). Sin embargo, se desconoce el motivo por el que se genera dismenorrea en EPI y en el ovario poliquístico (18).
El padecimiento data del tiempo de la menarca o poco después si los síntomas se iniciaron después de un año de la menarca, es necesario descartar presencia de padecimiento pélvico. El dolor se localiza en hipogastrio, en la región suprapúbica y se irradia algunas veces a la espalda o a la cara interna de los muslos. Puede iniciar unas horas antes de que se inicie la menstruación, pero en general se presenta una vez que el flujo menstrual ha aparecido. El dolor por lo general dura 24 a 48 horas y es raro que se prolongue por más de 72 horas. Es importante definir cómo es el dolor: si es cólico o continuo, y si llega o no 
a incapacitarla para sus actividades laborales y las situaciones o medicaciones con que cede.

La dismenorrea puede acompañarse de lumbago, náusea, vómito, diarrea, cefalea, mareo, cansancio, letargo, palidez o lipotimias pueden ser explicados por la secreción excesiva o el metabolismo escaso de prostaglandinas. Deben buscarse transtornos de la personalidad, psicosexuales, psicosomáticos y del carácter. Así mismo una relación entre la dismenorrea y otros síntomas que la orienten hacia una causa orgánica, por ejemplo: presencia de vulvovaginitis, ciclos anovulatorios y aunque la exploración física de la cavidad pélvica no se encuentran alteraciones en las pacientes con dismenorrea esencial, si pueden hallarse las malformaciones congénitas mencionadas; así como también definiría la presencia de masas anexiales o uterovaginales (12-13). Deben tomarse si se considera necesario todos los paraclínicos que puedan coadyuvar a un diagnóstico exacto (ecografía, histerosalpingografía, TAC, laparoscopia, etc.) (18).

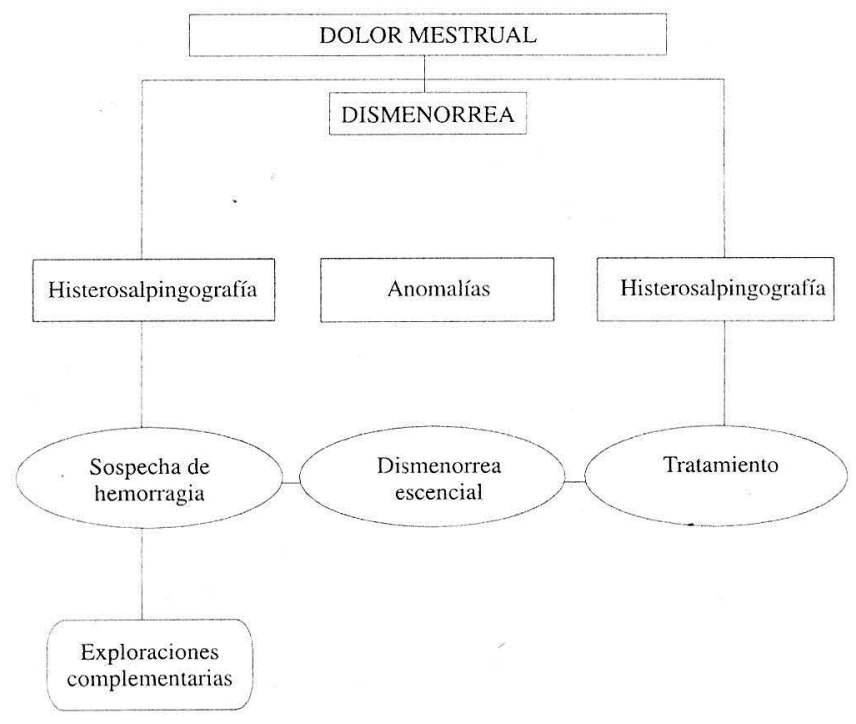

El problema del tratamiento sintomático de la dismenorrea esencial se mantuvo sin cambios durante muchos años. Pero desde que se descubrió que la elevación de los niveles de prostaglandinas son la causa de la dismenorrea, el tratamiento ha quedado bien definido.

Se precisa habilidad por parte del médico para descartar cualquier causa orgánica del padecimiento y dirigir a la paciente sobre su resolución las alternativas que aumentaron con la disponibilidad de un buen número de antiinflamatorios no esteroideos. (AINES). A medida que se fue conociendo la estructura y la acción de las prostaglandinas y conforme se estableció el mecanismo de acción de los AINES, se pudo confirmar el valor de este tipo de tratamiento. La eficacia de estos medicamentos puede deberse a su efecto analgésico directo o bien a la supresión de prostaglandinas el mecanismo de acción inhiben la vía de la cicloxigenasa, impidiendo la formación de endoperóxidos desde el ácido araquidónico o bien impidiendo la formación de PGE2 o PGF2 alfa desde el endoperóxido (19-21).
Los progestágenos reducen los niveles de prostaglandinas en el líquido menstrual a niveles inferiores de los normales, esta reducción se logra probablemente por dos mecanismos: supresión del tejido endometrial y por tanto del volumen del líquido menstrual y supresión de la ovulación, que produce ciclos anovulatorios con niveles de prostaglandinas semejantes a los de la fase proliferativa del ciclo normal, si no se quiere limitar o reducir la fertilidad o es preferible que la paciente no tome anticonceptivos orales, el fármaco de elección es un inhibidor de la síntesis de prostaglandinas, que sólo se administran durante la menstruación y no afectan la producción cíclica normal de hormonas (22-23).

Debe informarse a la paciente sobre su enfermedad, para esto se necesita de un médico comprensivo, dispuesto a dialogar con la paciente y prescribirle una medicación hasta que acepte su estado, enfrentaran pocos problemas o ninguno durante el período de reajuste de dolor (25-26).

Existen en Colombia, pocos trabajos sobre DISMENORREA EN LA ADOLESCENCIA uno de los más representativos fue el realizado por el Dr. Onatra W. y cols., (30), en 3 colegios de Santa Fe de Bogotá, y que nos servirá de marco de referencia. En el que encuestan a 496 adolescentes).

Estudios extranjeros señalan una incidencia variable del 45 al $80 \%$ y una tasa de ausentismo tanto del colegio como del trabajo del 3 al 22\% (31-33).

\section{Incidencia de la dismenorrea en adolescentes}

\begin{tabular}{|l|l|r|c|c|}
\hline Estudio & País & $\begin{array}{c}\text { No. de } \\
\text { casos }\end{array}$ & \% & Ausentismo \\
\hline Coppen 1963 & Inglaterra & 500 & 45 & $3-17$ \\
\hline Windholm 1971 & Finlandia & 3000 & 70 & 13 \\
\hline Sobczyk 1978 & E. Unidos & 113 & 89 & 20 \\
\hline Bjorn 1981 & Alemania & 656 & 72 & 15 \\
\hline Giordano 1891 & Brasil & 450 & 65 & 22 \\
\hline Onatra 1988 & Colombia & 496 & 76 & 14 \\
\hline
\end{tabular}

El promedio de edad de la menarquia es de 13 años (30, 34-35), la frecuencia del ciclo menstrual es de 28 por 4 días $(30,36)$. El dolor menstrual se presenta en el $67 \%$ de los casos antes de que los ciclos se hagan ovulatorios y mejoran al avanzar la edad (37-38) es mayor al inicio de la menstruación, siendo de intensidad leve en el 30$40 \%$ de las adolescentes, moderado en el 15-20\% y severo en un 3-15\% (33-36), en relación con el tratamiento mencionan la autoformulación y terapias no tradicionales con más frecuencia en aquellas que presentan dolor leve $(37-40,42)$.

No se mencionan las relaciones existentes con la actividad sexual de las adolescentes, la presencia de ciclos irregulares o patologías secundarias, no encontramos tampoco un estudio que presentara el seguimiento clínico de la adolescente (43).

El Fenoprofeno cálcico, derivado del ácido propiónico, cuya fórmula se indica en la figura. 


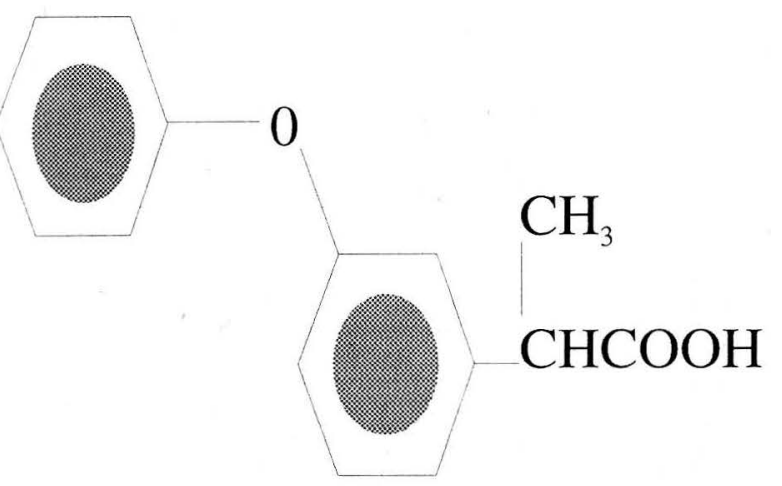

Es un antiinflamatorio con propiedades analgésicas y antipiréticas, es un inhibidor efectivo de la ciclooxigenasa responsable de la biosíntesis de las prostaglandinas.

Las dosis orales del fenoprofeno cálcico se absorben con rapidez $(85 \%)$, su concentración máxima en el plasma se alcanza después de dos horas y su efecto inicial a los 15 minutos.

\section{Material y métodos}

Diseño: Estudio abierto prospectivo controlado con placebo.

Se encuestaron 600 adolescentes pertenecientes al Colegio Departamental de la Merced j.t. de Mosquera. Fueron informadas con ayuda audiovisual sobre el origen, presentación, forma de aparición y demás aspectos que involucran el conocimiento práctico de la dismenorrea así como sus consecuencias y formas de tratamiento.

Las encuestas contaban con 7 variables dependientes y más de 50 variables independientes, realizadas a su vez como historia clínica de dismenorrea (anexo 1).

Se incluyeron en el estudio todas las adolescentes que refirieron padecer de dolor menstrual, entre 12 y 23 años de edad, con dismenorrea primaria de por lo menos tres ciclos de evolución.

Como criterios de exclusión se establecieron:

1. Antecedentes de ulcus péptico de cualquier orden.

2. Comprobación clínica de dismenorrea de origen secundario.

3. Aquellas con más de 24 horas de evolución de dolor dismenorreico o que hubiesen recibido medicación para ese episodio durante las 6 horas previas a su posible inclusión en el ensayo.

Se incluyeron 141 adolescentes, que sufrían de dismenorrea primaria con las que se formaron tres grupos. Según el medicamento que se les fuera a suministrar, así: 21 adolescentes con dismenorrea leve o moderada a las que se les trató con medios comunes tradicionales. El segundo grupo estuvo conformado por 35 adolescentes tratadas con placebo que presentaban dismenorrea de cualquier intensidad y el tercer grupo lo constituyeron 85 adolescentes tratadas con Fenoprofeno Cálcico.

Las 85 pacientes recibieron el primer día una dosis inicial de $600 \mathrm{mg}$ los dos días siguientes una cápsula T.I.D. Entre tanto el segundo grupo recibió el mismo número de tomas de un placebo y al tercer grupo se le administró un extracto aromático, en los tres casos el medicamento se administro durante tres días consecutivos. No se permitió el empleo de otros medicamentos que pudieran influir sobre el dolor o tuvieran un efecto sobre el sistema nervioso central, si así lo hicieron se retiraban del estudio.

Después de su absorción, el fenoprofeno cálcico se une casi por completo $(99 \%)$ a la albúmina plasmática. Se metaboliza en forma extensa (>90\%) y se excreta casi sin alteración por orina. Sufre la transformación metabólica al análogo 4-Hidroxifenoprofeno. Se forman cantidades casi iguales de conjugado con ácido glucoronico del mismo fenoprofeno y de 4-Hidroxifenoprofeno y juntos constituyen el $90 \%$ de la droga excretada, la vida plasmática es de alrededor de 3 horas.

El fenoprofeno cálcico se presenta en cápsulas de $300 \mathrm{mg}$.

Sus efectos secundarios son raros y están en relación con su actividad antiinflamatoria no. esteroidea.

\section{Análisis}

1. Se estudió la evolución de cada enferma durante 72 horas. Por 4 ciclos menstruales. Por 3 evaluadores supervisados por los autores. El dolor se cuantificó mediante escalas visuales de $100 \mathrm{~mm}$ de longitud, donde 0 correspondió a ausencia de dolor y el 100 al máximo del dolor, que tenían distribuido en su interior la graduación tácita del dolor para una mejor concepción de lo deseado por parte de los adolescentes. Esta escala se aplicó al momento de tomar las primeras dosis y al cabo de 15 y 30 minutos, 2 y 3 horas después, los días siguientes, se le pidió a la adolescente volver a evaluar su dolor (figura 1).

2. Se solicitó a la adolescente cuantificar e informar el tiempo que tardaba en sentir mejoría de su síntoma después de la ingesta del medicamento y con cada toma si presentaba dolor, dándole los siguientes parámetros:

Rápido: antes de 30 minutos.

Despacio: Antes de 2 horas y

No actuó: Si pasadas 3 horas no sentía mejoría de su sintomatología, en tal caso se le suministró terapias no tradicional como coadyuvante. Figura 2.

\section{Figura 1}

RELACION ENTRE EL NUMERO TOTAL DE ESTUDIANTES Y LAS QUE PADECEN

DISMENORREA, COL. DEPTAL LA MERCED

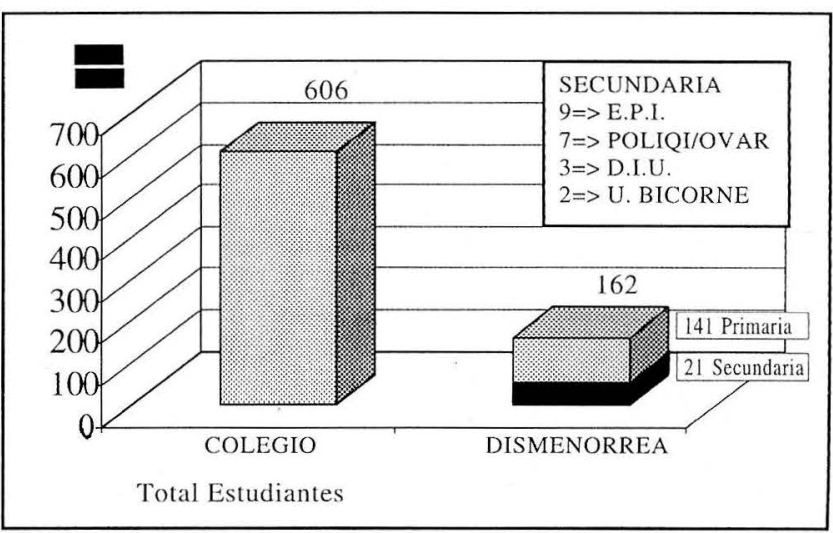


Figura 2

DISMENORREA EN ADOLESCENTES RELACION DE LA EDAD. COL. DEPTAL DE LA MERCED J.T. 1994

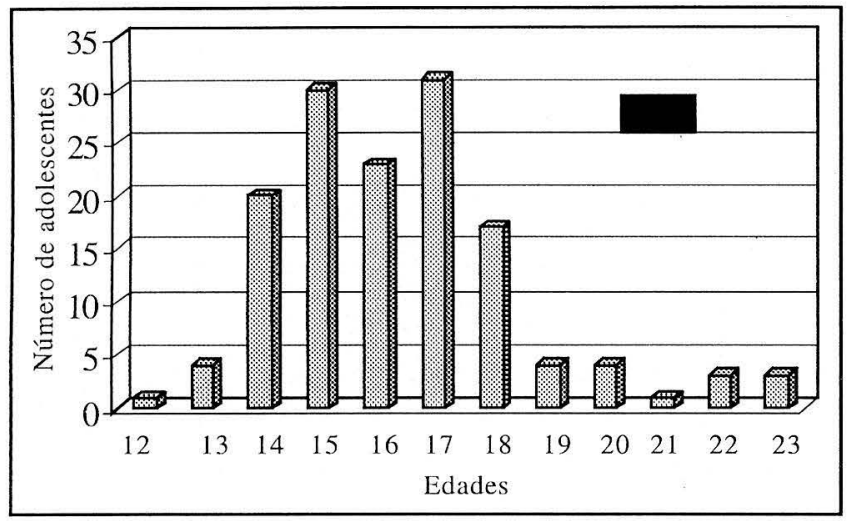

3. Así mismo se les solicitó evaluar el medicamento con base en la siguiente escala:

Bueno: Si mejoraba su sintomatología total y rápidamente.

Regular: Si presentaba después de las 3 primeras dosis dolor o si éste actuaba despacio.

Malo: Si presentaba dolor después de la $4^{\text {a }}$ dosis o si no actuaba.

4. Efectos secundarios: en las consultas posteriores, se interrogó sobre la presencia de efectos secundarios, al respecto se informó a cerca de:

1. Tipificación del síntoma.

2. Presentación horaria.

3. Intensidad.

4. Relación con la medicación.

Teniendo el resultado de lo anterior los investigadores decidieron la consulta a seguir según el siguiente protocolo:

a. No originado en el tratamiento, investigar o tratar el origen.

b. Relacionado con el medicamento: Suspender su administración.

c. Iniciar tratamiento de la patología secundaria.

5. Los investigadores opinaron sobre las terapéuticas instauradas calificándolas como:

Excelente, bueno, regular o inoperante.

\section{Resultados}

De las 600 estudiantes encuestadas el 27\% (162) padecían dismenorrea:

21 fueron diagnosticadas como dismenorrea secundaria, distribuidas así: 9 E.P.I. (Enfermedad Pélvica Inflamatoria), 7 Poliquistosis Ovárica, 3 D.I.U., 2. Malformación uterina, (Utero Bicorne). Comprobadas con los respectivos laboratorios clínicos y de imagenología. No se incluyeron en el estudio, iniciándose su tratamiento respectivo.

141 adolescentes fueron diagnosticadas como dismenorrea primaria, con ellas se inició el estudio, su ingreso fue progresivo.
E1 70\% de las adolescentes estaban entre los 15 y 17 años, su distribución según el grupo de trabajo: El promedio de edades de las que recibieron fenoprofeno cálcico de 16 años, para el placebo de 17 años y para la medicación no tradicional fue de 15 años.

El $60.99 \%$ de las adolescentes padecían dismenorrea desde la menarquia.

73/141 presentaban el dolor durante la menstruación, es decir desde el primer día de sangrado y los días consecutivos. 67/141 presentaban el dolor premenstrual sin afectar la época del sangrado, al contrario su aparición proveía mejoría espontánea de la sintomatología, sólo una refirió que el dolor iniciaba los últimos días de sangrado y persistía dos días más después del término.

El 36\% (51/141) manifestaron que el dolor se presentaba durante todo el día y era de carácter episódico por minutos, discrepando de lo contenido en la literatura revisada. $47 / 141$ (33.33\%) Refirieron que el dolor era predominantemente matinal cólico de duración regular por períodos de horas. En el 16\% fue de presentación vesperal y el $14 \%$ nocturno.

La duración del dolor en el $40,4 \%$ fue de dos días, en el $37,5 \%$ dura hasta tres días. En el $66,67 \%$ se presenta característicamente como de tipo cólico, 14/141 lo manifestaron como de tipo calambroide y 13/141 del tipo tiron, menos frecuentemente es de tipo presivo o picada.

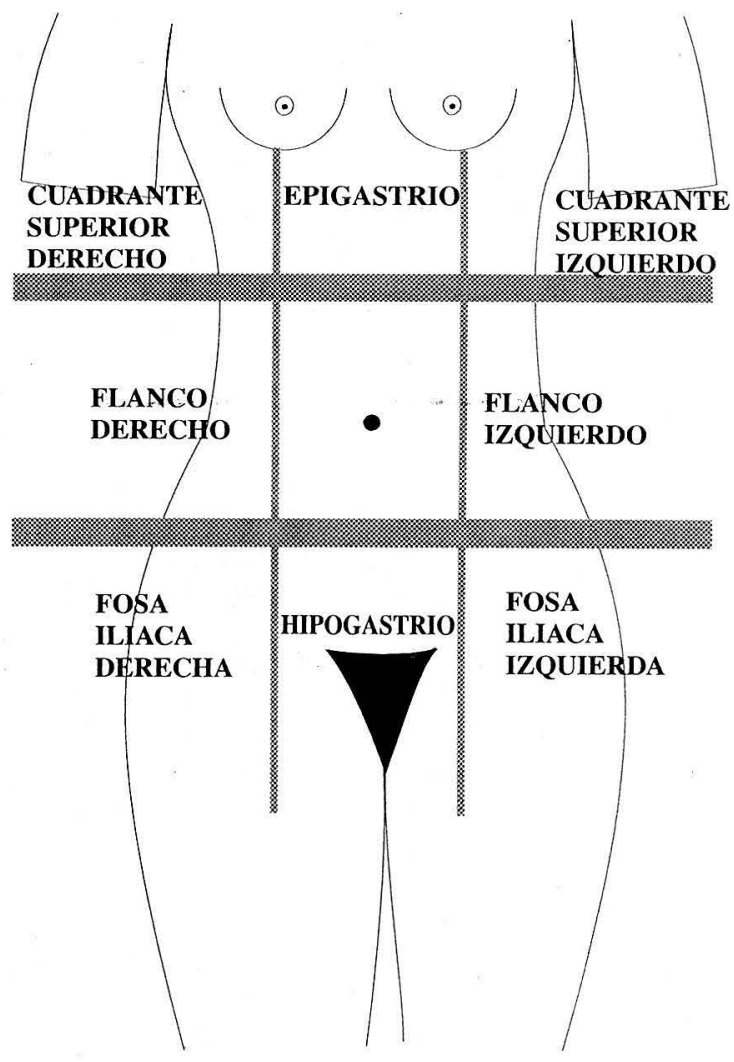


Según la distribución anatómica anterior, el 65\% de las adolescentes refirieron su dolor localizado en la región hipogástrica; en las fosas ilíacas y en la región umbilical localizaron el dolor 26/141 adolescentes en cada área $(78 / 141)$.

2.5 Síntomas asociados a la dismenorrea fueron referidos por cada adolescente, lo cual difiere de la literatura, por cuanto se menciona que su hallazgo es más frecuente a medida que aumenta la edad de la mujer, en orden de frecuencia, tenemos a la cefalea referida por el $50 \%$, malestar tipo peso en la región paralumbar $34,7 \%$; astenia (falta de ánimo para realizar las actividades cotidianas) $31,9 \%$; otros irritabilidad o carácter tendiente a el enfado más frecuentemente, disminución del rendimiento escolar, mareo, vómito, flatulencia.

El tiempo de evolución de la dismenorrea fue en promedio 2 años.

La graduación en escala del dolor mostró los siguientes resultados:

Grado I (leve) $27.66 \%$, Grado II (moderado) $54.61 \%$ y Grado III (Severo) $17.63 \%$.

Siendo más frecuente el dolor del orden moderado, justificamos esto como causa de automedicación y desconcierto personal ante la gama de terapéuticas que le son ofrecidas por unos y otros para aliviar su problema. Ya que el $36.8 \%$ de las adolescentes requieren por lo menos ciclo intermedio, de la ingesta de medicamentos para atenuar el dolor y el $31,9 \%$ lo ingieren siempre con cada ciclo menstrual.

Medidas alternativas o de medicina no tradicional utilizadas por las adolescentes para mejorar su sintomatología son:

Bolsa con agua caliente aplicada en hemiabdomen inferior $10 / 141(7 \%)$, reposo $27 / 141$ generalmente asociado al uso de la bolsa cálida, aguas aromáticas calientes y tomadas repetidamente de los tipos siguientes: Canela $16.31 \%$, Ruda 12,77\%, Yerbamora 15/141, Toronjil 12/ 141, menos frecuentemente: Manzanilla, Limón y Anís, o lilimento aplicado en el área de dolor.

Con relación a medicamentos el más frecuentemente ingerido para aliviar la sintomatología fue el Metil-etilbromure de eesina $12,06 \%$, A.I.N.E.S. como: Acido Mefenamico $9,9 \%$, Piroxican de $20 \mathrm{mg} 7.8 \%$, Ibuprofen $6,3 \%$, entre otros.

Las anteriores terapéuticas eran instauradas en el $35,21 \%$ de las adolescentes por un familiar, el $28,16 \%$ recurrían a los concejos de una farmaceuta (droguería); el $19,7 \%$ tomaban medicamentos sugeridos por compañeras u otras personas conocidas y sólo el 16,9\% obtuvieron la medicina por orientación de un facultativo; es decir que el $83,1 \%$ se automedicaban.

El $78 \%$ de las adolescentes se automedicaban no logrando mejoría de su sintomatología, ni con medicinas tradicionales, ni con las alopáticas, del $22 \%$ que obtuvo mejoría el $80 \%$ correspondió al grupo que consultó a un médico.

De las 141 adolescentes encuestadas, 33 tenían antecedentes de dismenorrea en su familia: 21/141 la(s) hermanas, 10/141 las madres y 2 las tías, 8 habían sido sometidas a apendicectomía entre 2 y 5 años atrás del diagnóstico.
61/141 adolescentes habían tenido relaciones sexuales, el $21 \%$ activas. De ellas planificaron en su momento o lo hacían actualmente 18,11 con anovulatorios y 15 con métodos de barrera ( 7 preservativos y 8 con tabletas vaginales).

La menarquia se presentó en el $52,48 \%$ de los casos entre los 10 y los 12 años y en el $42,5 \%$ entre los 13 y 14 años. No tenían ciclos menstruales regulares 79/141, entre las que el ciclo era regular $51 / 62$ se presentaban cada 30 días. La duración del sangrado en 85/141 adolescentes estaba entre 4 y 6 días; dura entre 1 y 3 días en 33/141 y sangraban por más de 7 días 23/141. En cuanto a la cantidad de sangrado teniendo como parámetro de medición el No. de toallas sanitarias/día, siendo abundante en 37/141 adolescentes, normal en 71/141 y escaso en 33/141.

Teniendo el consenso de que las toallas sanitarias vienen presentadas comercialmente en paquetes de aprox/ 10. $96 / 141$ refirieron utilizar un paquete por cada ciclo menstrual; 39/141 utilizan 2 paquetes y 7 tres paquetes.

Características al examen físico que interesan al ensayo fueron:

Relación Peso/talla/edad <- - > Peso/Talla, según las escalas del ICBF mostraron: 27/141 relación alta (sobrepeso); 17/141 relación baja (peso bajo para la edad y para la talla) y 97/141 figuran en la escala en los parámetros de normalidad que ellas indican. Se les dieron las indicaciones necesarias para que participaran en el programa de salud preventivo-curativo a que hubiera lugar, según el diagnóstico establecido llevado en la institución para que así solucionaran el evento que las acaecía.

Flujo vaginal se presentó en 33 adolescentes 26 de color blanco y características grumosas, en 19 amarillo mucoso, mal olor en 14 y prurito en 15 casos. Al examen 11 tenían edema de vulva e irritación local, en todos los casos no se practicó examen intravaginal, los exámenes paraclínicos concluyeron: 17 casos flujo fisiológico premenstrual concluyente por el no crecimiento de gérmenes en el cultivo practicado, 13 casos de Candidiasis Spp y 3 casos de Gardenerella Vaginalis. Todas las pacientes susceptibles a tratamiento farmacológico ingresaron al programa institucional de flujo vaginal en el que recibieron los medicamentos necesarios para su tratamiento y la orientación propia del programa (XVIII Congreso Colombiano de Pediatría, Memorias, Sociedad Colombiana de pediatría Sep./1993).

El tratamiento se distribuyó en 4 ciclos menstruales y en cada uno se evaluó la respuesta sintomática al tratamiento instaurado, en el cuarto ciclo no se suministro tratamiento se le hizo el seguimiento, según la escala visual propuesta.

Los valores promedios del dolor expresados en mm, obtenidos en las escalas visuales las presentamos en diagramas comparativos (figuras 3-7) donde se demuestra que después de la primera dosis, existe una clara diferencia, muy significativa, en favor del fenoprofeno cálcico, la cual se mantuvo a todo lo largo del período de observación, con una p $<0=0.01$ (es decir su nivel de confianza es del 99\%) al compararlo con el placebo y con el medicamento natural. 
Las curvas de objeto de la observación están compuestas en general por dos partes: la acción rápida después de la primera dosis lo mismo que la mejoría franca del dolor en la mayoría de las adolescentes y la evolución tendiente a la disminución del síntoma durante los siguientes días de tratamiento en los días subsiguientes.

Se presenta la relación de los diferentes medicamentos durante el primer ciclo de tratamiento, donde el fenoprofeno ofrece una mejoría a más del $80 \%$ de las adolescentes en forma rápida, es decir antes de 15 minutos.

Se presentan la misma relación durante el tercer y cuarto ciclo de evaluación, al final del ensayo el fenoprofeno dio mejoría al 97,5\% de las adolescentes.

Figura 3

DISTRIBUCION DE LAS PACIENTES CON DISMENORREA SEGUN SU INICIO EN RELACION A LA MENARQUIA COLEGIO DEPTAL LA MERCED J.T. 1994

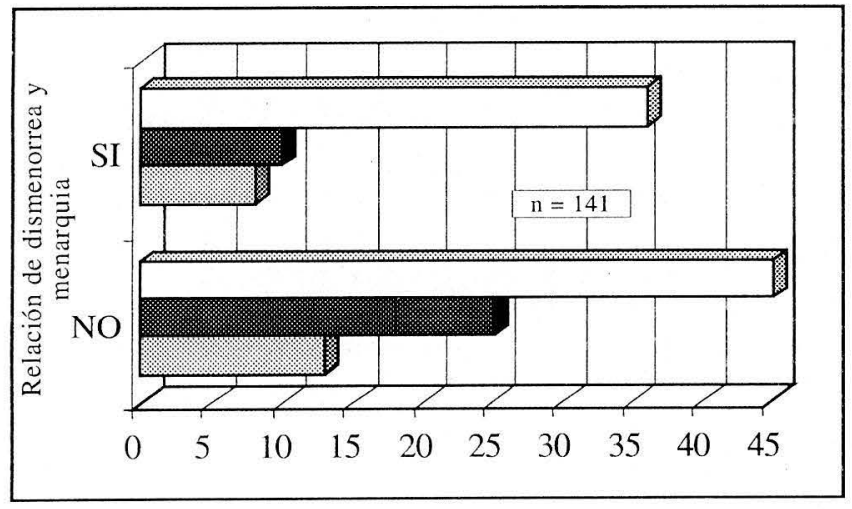

Figura 4

RELACION DEL SANGRADO MENSTRUAL Y LA PRESENCIA DE DISMENORREA EN ADOLESCENTE COLEGIO DEPARTAMENTAL DE LA MERCED J.T. 1994

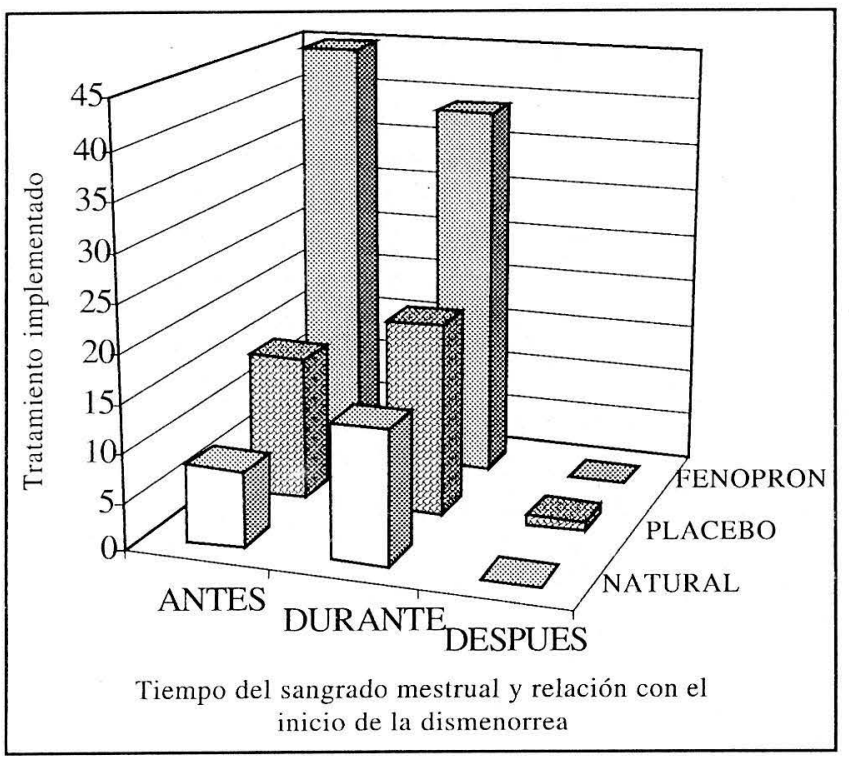

Figura 5

DURACION DEL DOLOR MENSTRUAL EN ADOLESCENTES CON DISMENORREA EN EL COLEGIO DEPARTAMENTAL DE LA MERCED J.T. MOSQUERA CUNDINAMARCA, 1994

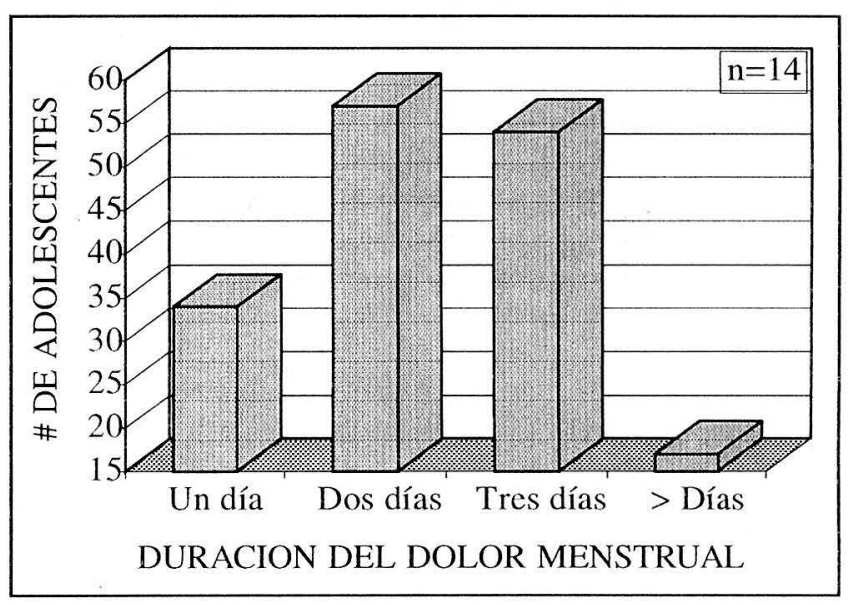

Figura 6

NECESIDAD DE MEDICACION EN ADOLESCENTES CON DISMENORREA, COL. DEPTAL DE LA MERCED J.T. MOSQUERA, CUNDINAMARCA. 1994

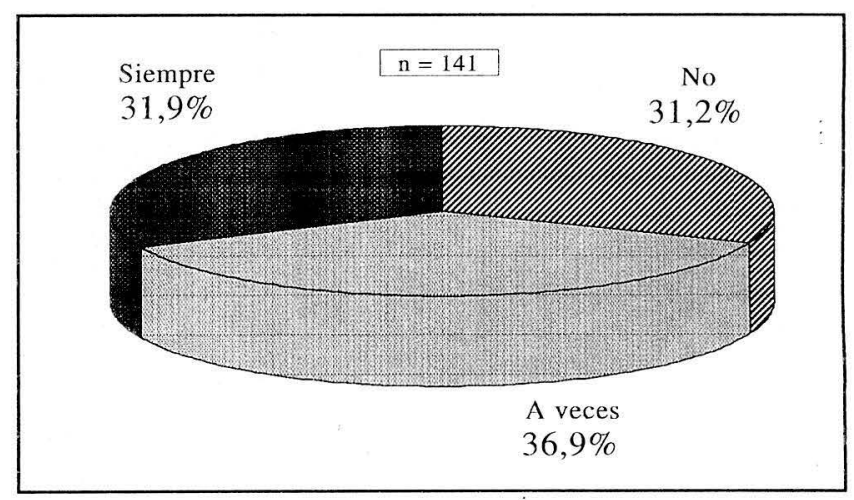

Figura 7

ESCALA VISUAL DEL DOLOR. DISMENORREA EN ADOLESCENTES

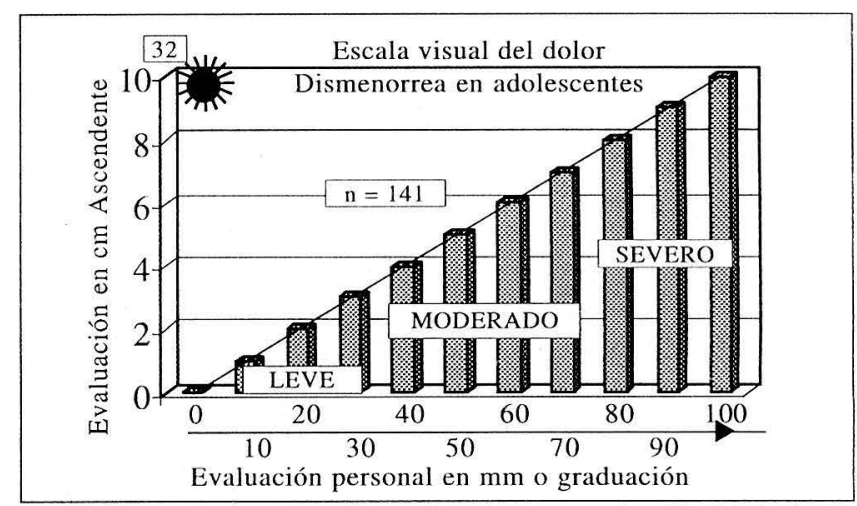


Al final del primer período de observación, la curva de la escala visual presenta el nivel del dolor para fenoprofeno cálcico significativamente inferior a el placebo $(\mathrm{p}<=0.01)$. Esta diferencia expresada en porcentaje de remisión del dolor, es la siguiente: Con el placebo, el dolor se redujo a las 3 horas en el $32 \%$, mientras que con el fenoprofeno cálcico, la disminución fue de $88 \%$. Durante el segundo día, la curva también ilustra cómo evolucionó el dolor en forma igualmente favorable para el fenoprofeno cálcico.

Así expuesto la diferencia continuó constante hasta el tercer día, y durante cada ciclo de tratamiento hasta la evaluación global o cuarto ciclo, en el cual el dolor era de intensidad leve.

Se describe la utilidad del fenoprofeno cálcico en la dismenorrea de las adolescentes hasta el 3er ciclo de tratamiento, demuestra la mejoría global del $97 \%$ (total: $89 \%$ y bastante $8 \%$ ).

La acción del fenoprofeno cálcico se presenta y se concluye que mejora rápidamente (antes de 30 minutos) al $92.94 \%$ de las adolescentes.

En general el $96 \%$ de las estudiantes tratadas con fenoprofeno cálcico opinaron que este medicamento es bueno, les alivia rápido y totalmente su sintomatología.

Se compara al placebo y al fenoprofeno en todo orden de análisis objeto del estudio, la evaluación de la p es $<0,001$ a favor del fenoprofeno, lo cual es estadísticamente significativo.

La relación porcentual de la rapidez de acción de los medicamentos estudiados se presenta en el fenoprofeno que actúa entre 15 y 30 minutos en el $95.29 \%$ de las adolescentes al tercer ciclo de tratamiento, al compararlo con el $82 \%$ del placebo y el $81 \%$ de la medicina alternativa, demuestra a favor del fenoprofeno una mayor rapidez de acción.

Se presentan los resultados globales a orden porcentual del tratamiento de la dismenorrea en adolescentes.

\section{Efectos secundarios}

Con el fenoprofeno cálcico se presentaron efectos secundarios en 4 pacientes. Un caso curso con dispepsia que duro 3 días y requirió de medicación alterna. Otra paciente tuvo pirosis que cedió a la administración de gel de hidróxido de aluminio y se considero consecuencia del medicamento. Epigastralgia tipo ardor que cedió espontáneamente al suspender el tratamiento. Con el placebo se presentaron como efectos secundarios dos casos de náusea, un caso de dispepsia, dos pacientes con pirosis una de las cuales con sensación de reflujo que requirieron de tratamiento además de dos casos de epigastralgia. Con la medicación natural se presentaron tres casos de náusea y un caso de vómito, dos casos de dispepsia. Resultados estadísticamente no significativos.

Obteniendo la mediana de cada uno de los aspectos investigados el fenoprofeno cálcico en comparación con el placebo y la medicina natural permite aún más confirmar la eficacia del fenopron. Se presentan las curvas estadísticas de la relación porcentual del tratamiento de la dismenorrea en adolescentes y sus resultados.
La opinión de los investigadores respecto a la eficiencia del medicamento al final del período de observación fue la siguiente: El fenoprofeno cálcico fue excelente o bueno en el $95 \%$ y regular o nulo en el $5 \%$ por lo que respecta al placebo, opinamos que fue bueno o excelente en el $40 \%$ y nulo en el $60 \%$. La diferencia de los resultados obtenidos con fenoprofeno cálcico y placebo, favorece al fenoprofeno cálcico y tiene significación estadística de $\mathrm{p}<=0.01$.

\section{Discusión}

La dismenorrea es un problema frecuente de las adolescentes, para nuestra institución constituye la tercera causa de morbilidad estudiantil, después del parasitismo intestinal y los problemas de visión, según resultados de la evaluación de morbilidad 1992-1994. La padecen el $27 \%$ de las estudiantes $(162 / 606)$, el $12 \%$ de ellas (21/ 162) padecen de dismenorrea secundaria, según lo descrito anteriormente, siendo la entidad causal más frecuente la enfermedad pélvica inflamatoria $9 / 21$ y en segunda instancia la poliquistosis ovárica, a estas estudiantes se les proporcionó el tratamiento propio de su patología y no se incluyen dentro del estudio.

El promedio general de la edad de las 141 adolescentes evaluadas era de 14,6 años. El 59\% de las adolescentes con dolor menstrual tienen entre 15 y 17 años, y hecho en relación a la literatura mundial. Se presenta más frecuentemente durante la menstruación y por lo general después del primer día de sangrado situación que no concuerda con lo descrito en los ensayos analizados donde se infiere como más frecuente la presentación premenstrual.

La dismenorrea se presenta con mayor frecuencia a lo largo del día, durante dos días en promedio (77\%), variando entre uno y 8 días, se presenta en episodios de dolor tipo cólico que duran entre 10 y 35 minutos, en períodos de ascenso, meseta y lo localizan con mayor frecuencia a nivel de hipogastrio y no en las fosas ilíacas como aparentemente es lo más frecuente según los diferentes estudios publicados.

La graduación del dolor en 3 categorías y éstas a su vez en subclases permite identificar claramente, con ayuda de las escalas visuales, la intensidad del dolor, que para nuestro caso el dolor leve corresponde al $27,66 \%$, el moderado en el $54.61 \%$ de las adolescentes no relacionado con lo descrito por Onatra W. y cols., donde el resultado es inverso. El $17.63 \%$ (25/141) de las pacientes presentaban dolor severo de las cuales el $39 \%$ era incapacitante (10/25), resultado también no relacionado con lo descrito anteriormente donde se establece que el dolor incapacitante lo presenta entre el $1,6 \%$ y $3 \%$ de las adolescentes, en nuestro ensayo corresponde al 7\%.

Además de la dismenorrea las adolescentes describen otros síntomas asociados con su ciclo menstrual, establecimos, sí, que se tratara de presentación cíclica bien cualificada. El 50\% refiere cefalea global inespecífica asociada a la menstruación, otros síntomas referidos son astenia, irritabilidad, y disminución del rendimiento escolar, puede configurarse en algunas de ellas inclusive un síndrome de tensión premenstrual. 
El $68 \%$ de las adolescentes estudiadas recurren a medicaciones diversas en el intento de mitigar el dolor dismenorreico, a diferencia del estudio de Onatra y cols, las que con mayor frecuencia toman algún medicamento son las que padecen de dolor moderado o severo; el $41 \%$ se automedica por consejo de familiares, conocidos o farmaceutas, situación de por sí generadora de otras patologías de índole iatrogénico y consumista, acostumbrando a las personas desde jóvenes a ingerir toda índole de medicamentos por su cuenta y riesgo sin tener en cuenta los riesgos futuros de tal comportamiento. Toman diferentes tipos de antiinflamatorios no esteroideos, espasmolíticos y analgésicos simples; además de 8 diferentes clases de aguas aromáticas, los anteriores, tanto medicamentos como medidas alternativas sólo proporcionan mejoría total al $36 \%$ de las adolescentes que los ingieren y parcial al $67 \%$ del total, otra pauta que permite establecer lo mal orientado del tratamiento de la dismenorrea en la adolescencia, aún más al comprobar que sólo el $8 \%$ de ellas consultan al médico por su problema.

Concluimos que debe permitirse desde el aula una orientación adecuada respecto al tratamiento de esta patología, agrupándola dentro de los programas institucionales de salud en los diferentes colegios que cuenten con servicio médico.

Solamente el $23 \%$ de las adolescentes tienen antecedentes familiares de dismenorrea no siendo esto estadísticamente significativo, no tenemos referencias de estudios al respecto. Al igual que la actividad sexual positiva en el $26 \%$ del total y activa en el $12 \%$, tampoco parece tener influencia sobre el padecimiento aún las que planificaban con anovulatorios orales padecían de igual intensidad la dismenorrea.

La historia ginecológica nos muestra que la menarquia en el 54\% se presentó antes de los 12 años, entre 1 y 3 años antes de lo descrito en la literatura mundial. En relación con el ciclo menstrual se presenta con frecuen- cia irregular entre 25 y 30 días. Durante 4 y 6 días en cantidad normal según lo considerado por la propia adolescente resultados de acuerdo con lo descrito por Onatra y cols y el estudio de la OMS a que hacen referencia.

El seguimiento del ciclo menstrual durante los cuatro episodios controlando el tratamiento, nos permite evaluar la validez de los tratamientos más comunes tanto de las medidas alternativas como un antiinflamatorio no esteroideo; no utilizado comúnmente por las adolescentes, controlando los resultados con un grupo placebo.

Los resultados por ciclo están bien descritos anteriormente. El promedio general obtenido por el análisis de la mediana estadística se aprecia en las figuras 4-7. Donde se demuestra que utilizando correctamente el AINE (Fenoprofeno cálcico) mejora al $96 \%$ de las adolescentes tratadas, además de que a partir del tercer ciclo disminuye significativamente la intensidad y frecuencia del dolor menstrual, así como las manifestaciones clínicas asociadas al ciclo menstrual, tales como cefalea, astenia, náusea y otras más, comparándolo con el placebo que dio mejoría al $78 \%$ la diferencia estadística es significativa.

Las medidas tradicionales como reposo, bolsa con agua caliente y medicamentos de origen natural mejoran al $75 \%$ pero no disminuyen la frecuencia del dolor ni su intensidad en los ciclos subsiguientes.

La rapidez en cuanto al control del dolor está a favor del fenoprofeno cálcico en una relación de 2: 1 tanto del placebo como de la medicación natural. Actúa entre los 10 y 25 minutos después de ingerido el antiinflamatorio.

La opinión de las adolescentes en relación a la efectividad del tratamiento y el medicamento en general es buena para el $90 \%$ en la mediana estadística para el fenoprofeno cálcico, 43\% para el placebo y 33\% para la medicina alternativa.

Los efectos secundarios presentados no son estadísticamente significativos.

\section{BIBLIOGRAFIA}

1. Dawood MY. Choosing the corret therapy for dysmenorrhea. Contemp Ob. Gyn. 1982; 19: 235 .

2. Warren TK. Dismenorrea. Mundo Médico, 1988; 4(1): 45-46.

3. Cabrero L. Dismenorrea. Masson-Salvat, 1993. Capítulo 8.

4. Woodbury R. Myometrial phisiology and its relation to pelvic pain JAMA 1947; 134: 1081 .

5. Ylikovkala O. New concepts of dysmenorrhea. Am. J. Obstet. Gynec. 1978; 130: 833 .

6. Cleary R. Dysmenorrhea. In Current therapy in obstetrics and gynecology. Philadelphia. Saunders Co., 1983; 150.

7. Gantt P. Dismenorrea en pediatría. Clínicas Pediátricas N. Am. 1981; 2: 387 .

8. Moir J. Intrinsic Dysmenorrhea. Proc. R. Soc. Med. 1976; 29: 850.

10. Pikles VR. Prostaglandins in human endometrium Internal. J. fertil. 1967; 12: 335 .

11. Dickins A. Dismenorrea, Ginecología y Obstetricia. Temas actuales $1974 ; 656$.

12. Hillier $\mathrm{H}$. Prostaglandin F 2 Alfa administrations in luteal phase of the menstrual cycle. Brit. Med. J. 1972; 4: 333.

13. Jones $G$. The effect of prostaglandin F 2 alfa infusium en corpus luteum function. Am. J. Obstet. Gynecol. Scand. 1971; 14: 1.
14. Akerlund M. Primary dysmenorrhea and vasopresin. Br. J. Obstet. Gynecol. 1979; 36: 484.

15. Gautier PL. Endometriose de la jeune fille. Rapports III éme. Symposium International sur la Gynecologie de l'efant et del l'adolescente. Lausanne 1976; 207.

16. Goldstein D. Adolescent endometriosis. J. Adolescent Health Care 1980; 1: 37.

17. Ossev $\mathrm{S}$. Pisk of pelvic inflamatory disease among use of intrauterine devices, irrespective of previus pregnancy. Am. J. Obstet. Gynec. 1968; 138: 864.

18. Goldstein D. Laparoscopy in the diagnosis and management of pelvic pain in adolescents. J. Reprod. Med. 1980; 24: 251.

19. Kantero R. Correlation of menstrual trains between adolescent girls and their mothers. Acta. Obstet. Gynecol. Scand. 1971; 14: 1.

20. Henzl M. The treatment of dysmenorrhea. Am. J. Obstet. Gynec. 1977; 127: 818

21. Lundstrom $V$. Treatment dysmenorrhea primary winth prostaglandin synthetase inhibitors: A promising therapeutic alternative. Acta. Obstet. Gynecol. Scand. 1978; 57: 421.

22. Duarte-C. Endometrio en ciclos libres posterapia ovulostática administrada a largo plazo. Rev. Colomb. Obstet. Ginecol. 1968; 19: 3. 
23. Widholm A. Menstrual patterns of adolescent girls according to chronological and gynecological ages. Acta. Obstet. Gynecol. Scand. 1971; 50: 19 .

30. Onatra W. Dismenorrea en adolescentes: Estudio epidemiológico. Memorias del curso. Complicaciones médico-quirúrgicas en ginecología. Universidad Nacional. Agosto de 1989.

31. Sobczyk R. Dysmenorrhea in the family practique population. J. Farm. Pract. 1978; 7: 285.

32. Giordano J. Estudio sobre dismenorrea en mujeres entre 18-40 años. Traducción Hospital 1983; 2: 38.

33. Who. Health organization Multicenter study of menstrual and ovulatory patterns in adolescent girls. J. Adolescent Health Care. 1986; 7: 236.

34. Ucros-Cuellar. El adolescente en Colombia: Variables fisiológicas del desarrollo pondo-estatural y sexual. Medicina del adolescente. Colegio Mayor de Nuestra Señora del Rosario.
35. Jaramillo R. Adolescencia. Rev. Col. Obstet. Ginecol. 1984; 35: 401.

36. Marshall W. Variations in pattern of puberal changes in girls. Arch. Dis. Child. 1989; 44: 291.

37. Winlholm O. Correlations of menstrual trais between adolescent girls and their mothers. Acta. Obstet. Gynecol. Scand. 1971; 50: 30.

38. Wilson C. The relationships of calculated percent body fat, sports participation, age, and place of residence on menstrual patterns in healthy adolescent girls at an independent New England High School. Sexual. Activ. Teenagers. 1988; 2: 163.

39. Pizarro E. Estudio Comparativo sobre el efecto del DIU progestasert y gravigard en la dismenorrea. Contracepción. 1979; 20: 455.

40. Huffman H. Dysmenorrhea premenstrual tension and endometriosis. In the gynecology of childhood and adolescence. Philadelphia: W.B. Saunders Co. 1981

41. Larkin R. Dysmenorrhea. Obstet. Gynecol. 1979; 54: 456. 